\title{
Innovative Coatings for Tooling, Machining, Aerospace, Energy and Automotive Industry
}

\author{
Paul Mayrhofer \\ Institute of Materials Science and Technology, Technische Universiaet Wien \\ Karlsplatz 13, 1040 Wien \\ Austria \\ paul.mayrhofer@tuwien.ac.at
}

This work summarizes recent developments on applying thin film structure and architecture concepts to hard coatings for optimized performance in various application fields. Hard coatings deposited by plasma-assisted vapour deposition are widely used to reduce friction and wear of tools and engineering components in energy, automotive and aerospace industry.

We will look in more detail into the correlation between microstructure and mechanical and thermal properties of hard ceramic coatings. This is done for single-phase coatings and composition or phase modulated layers. In the latter case, the microstructure can be designed by choice of the deposition technique, understanding the growth processes taking place on a film surface, either by sequential deposition of layers or by taking advantage of self-organization processes including segregation effects of the elements. Consequently, the effects of individual microstructural features like grain size, defect density (and hence residual stress), phase arrangements in an one-, two- or three-dimensional manner on the mechanical properties are treated.

The microstructural changes of hard ceramic coatings during a post-deposition annealing treatment are discussed in detail. Although the significance of heat treatments to optimize properties (by a well-designed microstructure) for specific applications is recognized in bulk material science, only a few elements have been applied for hard ceramic coatings so far. The various thin film structure and architecture concepts allow the utilization of multifunctional properties facilitating the development of next generation's hard coatings.

\section{References:}

[1] P. H. Mayrhofer, C. Mitterer, L. Hultman, and H. Clemens "Microstructural design of hard coatings," Prog. Mater. Sci. vol. 51, pp. 1032-1114, 2006. 